\title{
THE COMPARISON OF THYRIOD HORMONES LEVELS IN BLOOD BETWEEN LOCAL CHICKEN AND BROILER
}

\author{
AYHAM MOHAMMAD ABDULKADER; AMER DABBAG and HASSAN ALKRAD \\ Department of Animal Production, Faculty of Veterinary Medicine, Hama University
}

\author{
Received: 17 July 2016; $\quad$ Accepted: 31 August 2016
}

\begin{abstract}
A total of 300 one day old chicks (150 broiler chicks, 150 local Syrian chicks) were raised for 6 weeks in order to measure blood concentration of Thyroxine and Triiodothyronine T3, there were 2 treatments 3 replication for each. At the end of every week 6 birds were weighed and bled in order to examine thyroid hormones. The results showed that the local chicken had higher thyroid hormone concentration than broiler at all ages $p \leq 0.001$. At the age of one week the level of T4 and T3 were $10.31,1.81 \mathrm{ng} / \mathrm{ml}$ respectively for local chicken and $6.5,0.9 \mathrm{ng} / \mathrm{ml}$ for broiler. While the level of both hormones continue to drop all over the experiment to reach at the age of 6 weeks in local chicken 7,1.29 $\mathrm{ng} / \mathrm{ml}$ for T4 and T3 respectively and 5.5,0.54 in broiler with strong correlation between weight age and both hormone levels, but the relation where stronger in local than broiler. Our result suggest that the genetic selection over time for increased meat production induced metabolic shift and thyroid hormone level in one of its outcome.
\end{abstract}

Key words: Broiler, Local chicken, T4,T3, Live body weight.

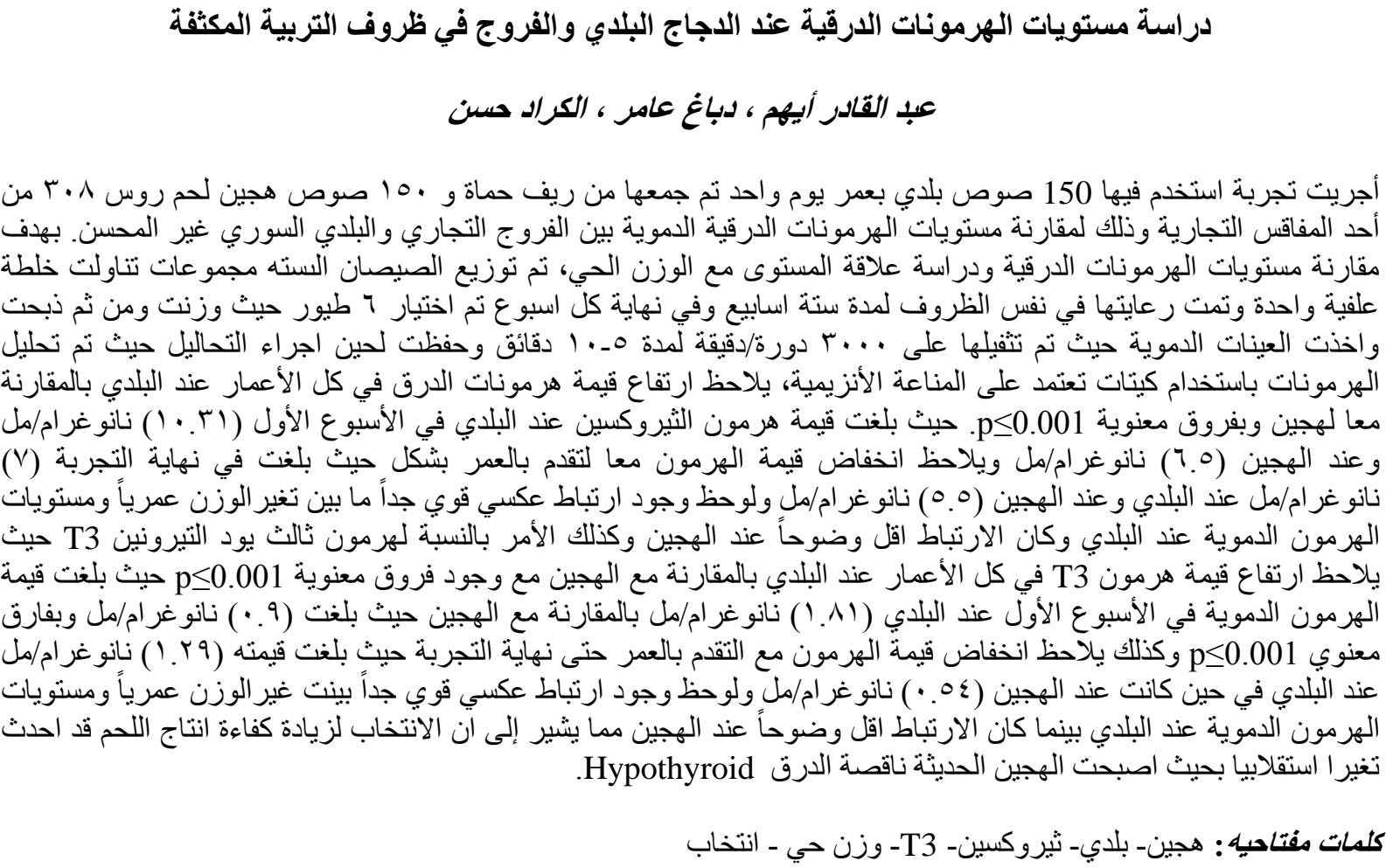

Corresponding author: Dr. AYHAM MOHAMMAD ABDULKADER

E-mail address: dr.ayhamabdulkader@yahoo.com

Present address: Department of Animal Production, Faculty of Veterinary Medicine, Hama University 


\section{INTRODUCTION}

\section{المقدمـــــة}

لقد حدث تطور هائل في المقدرة الإنتاجية لسلالات الفروج الحديث وانتج الانتخاب الوراتي تغييرات في استقلاب وفيزيولوجيا

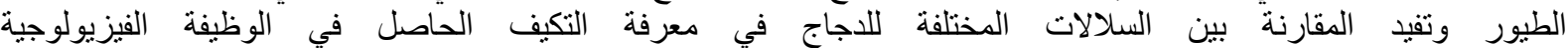
(Scanes, 2011) بنفس التركيب الكيميائي عند كل الفقاريات بما فيها الطيور والثييات (Thiiodothyrionine-T3

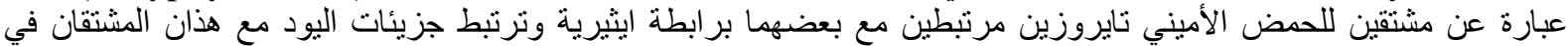

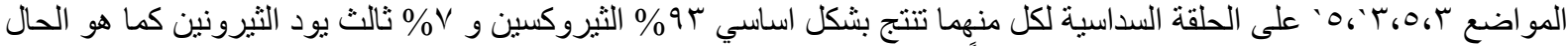

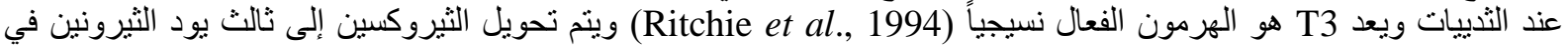

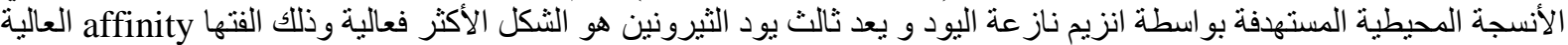
للمستقبلات الخلوية مقارنة بالتيروكسين.

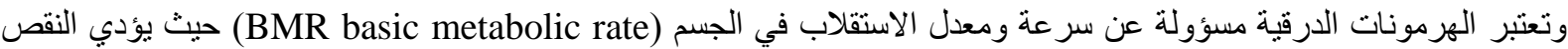

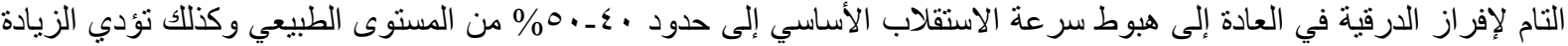

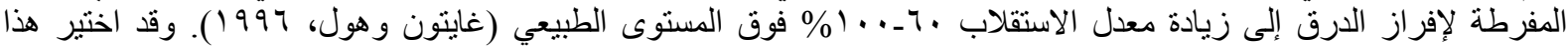

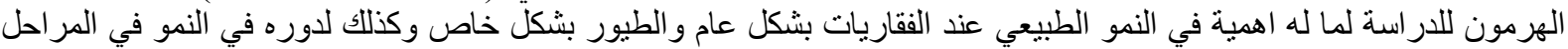

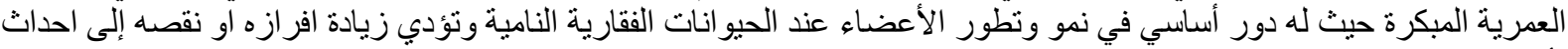

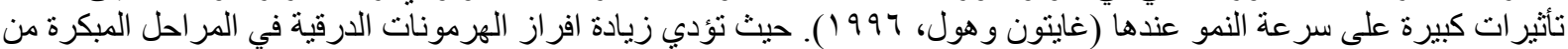

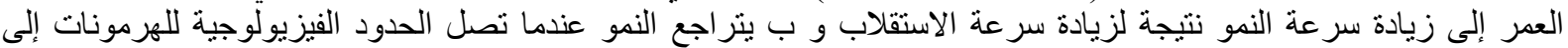

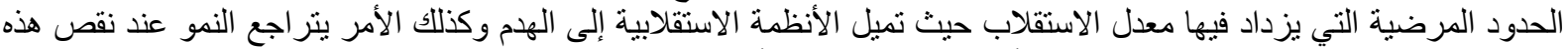

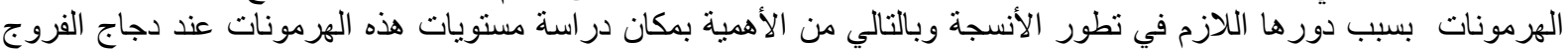

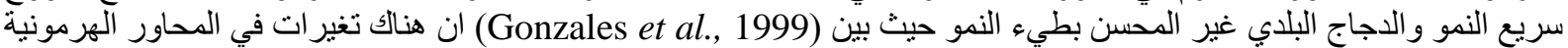
الاستقلابية رافقت التحسين الور الثي باتجاه زيأدة سر عة النمو وزيادة وزن الجسم.

\section{MATERIALS AND METHODS

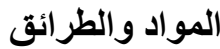

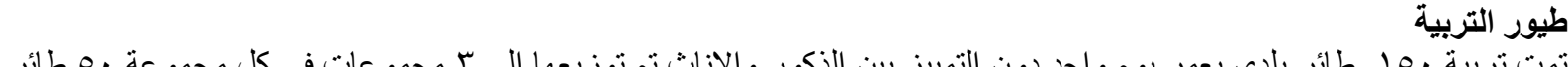

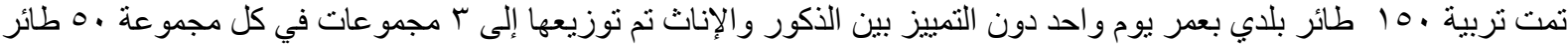

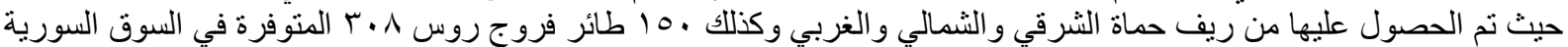

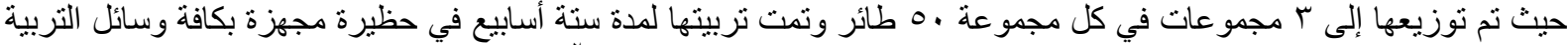

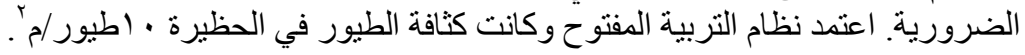

في عمر سبعة أيام تم إعطاء لقاح مشترك لمرض النيوكاسل (ND) و التهاب القصبات المعدي B1) + H120 من طريق العين تم

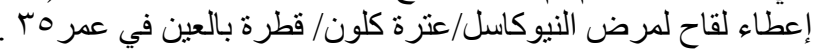

قسمت فترة التربية إلى مرحلتين: المرحلة الأولى ( من ا إلى (r يوم ) و المرحلة الثانية ( من بr إلى إك يوم). الجدول رقم (1) يبين تركيب الخلطة المستخدمة في التجربة و الجدول رقم ( ب) يبين القيم الغذائية لهذه الخلطات.

وزنت طيور كل مجمو عة بشكل أسبوعي وفي نهاية كل اسبوع تم اختيار 7 طيور عشو ائياً من كل مجموعة وسجل الوزن الحي لكل

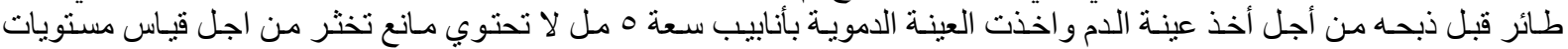

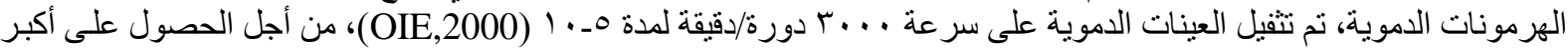

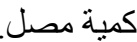

قياس الهرمونات الدرقية:

Competitive Enzyme Immune assay - بم قياس مستويات الهرمونات الدرفية T3 و T4 باستخدام المقايسة المناعية الأنزيمة . EIA

قياس هرمون الثيروكسين

تم استخدام مجموعة تشخيصية خاصة مصنعة من فبل شركة TOSOH وتدعى المجموعة التشخيصية ST AIA-PACK T4 للقياس الكمي لهرمون الثيروكسين في المصل او البلازما المهبرنة. 
ميتعلومات هذا الكيت: لكيث لقياس تركيز هرمون الثيروكسين في عينات المصل او البلازما المهبرنة باستخدام جهاز التحليل الأوتوماتيكي TOSOH AIA SYSTEM Analyzers

ملخص عن الإختبار يعتبر تقييم الحالة الدرقية عملية معنية معقدة ، و الوظيفة الرئيسية للغدة الدرقية هي انتاج وافراز هرمون الثيروكسينT4 وثالث يود

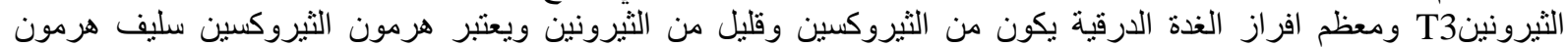
الثيرونين الأكثر فعالية حيث يعتبر الإنتاج الأعظمي للثيرونين خارج الدرقية في الأنسجة المحيطية بواسطة الأنزيمات نازينة عة اليونين

مبدأ المقايسة تعتبر هذه المقايسة أنزيمية تنافسية Competitive enzyme immunoassay وتجرى بشكل كامل في الأكو اب الخاصة المرفقة

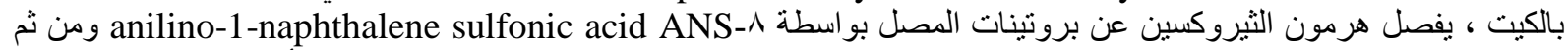

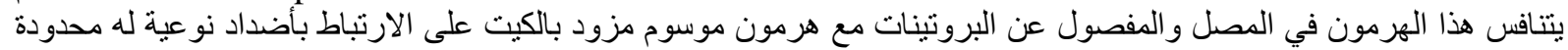

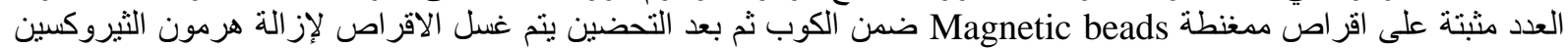
الموسوم غير المرتبط ويتم تحضين هذه الأقراص بركيزة منتجة للضوء (phosphate (4MUP ، ونتناسب كمية الثيروكسين الموسوم المرنبط مع الأضداد عكسياً مع تركيز الثيروكسين الموجود في في العينة ويتم رسم منحنى بياني بالإعتماد على المحاليل المعيارية المزودة مع الكيت وبالمقارنة معها يتم حساب تركيز الثين تركيز الثيروكسين في عينة

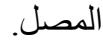

المواد المقدمة مع الكيت كوربت

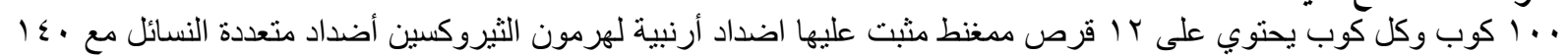

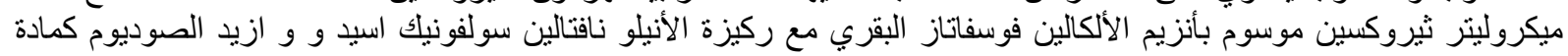

حافظة.

قياس هرمون ثالث يود الثيرونين T3

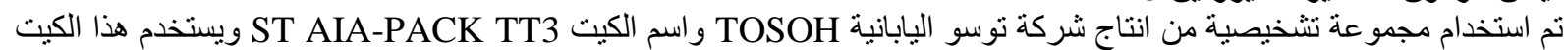

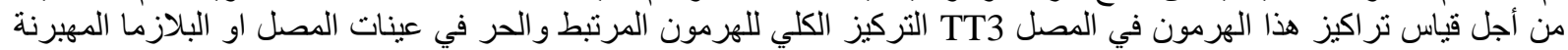

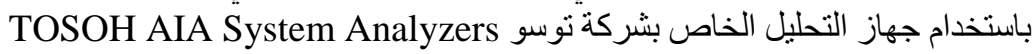

و هو عبارٔ الإختبار عن مقايسة أنزيمية مناعية تنافسية Competitive Enzymatic Immunoassay و التي تجرى بشكل كامل في الأكو اب المزودة مع الكيت.

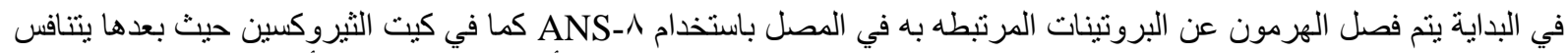

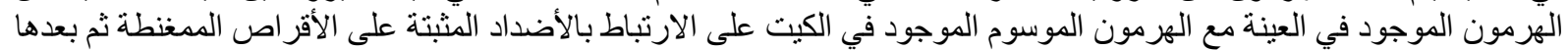

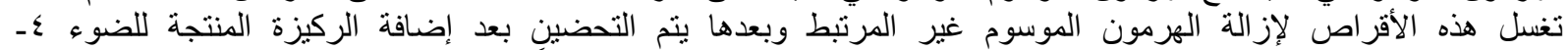

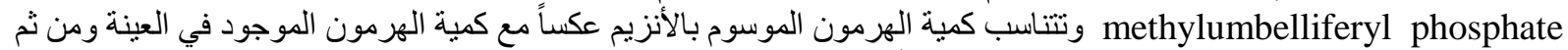
يتم انتاج منحنى فياسي بو اسطة المحاليل العيارية التي أجريت مع الاختبار ويتم حساب تركيز الهرمون في العينة بالاعتماد عليها و الاستجابة اللونية الناتجة عن العينة في نهاية الاختبار.

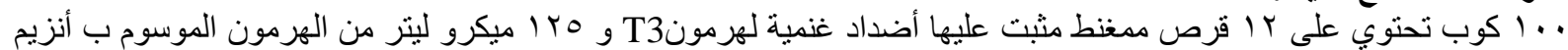

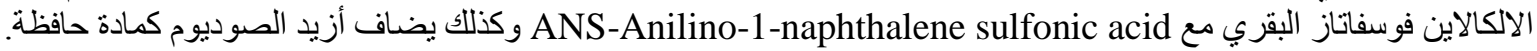

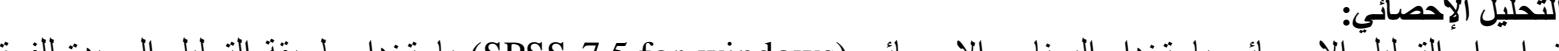
تم إجراء التحليل الإحصائي باستخدام البرنامج الإحصائي (SPSS 7.5,for windows) باستخدام طريقة التحليل الوحيدة للفرق ANOVA (One-Way Analysis Of Variance)

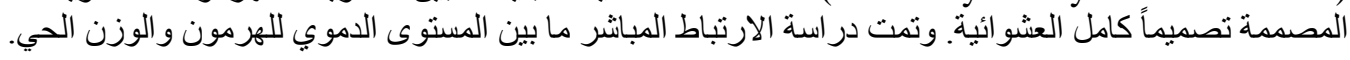

اولا لأنتائج

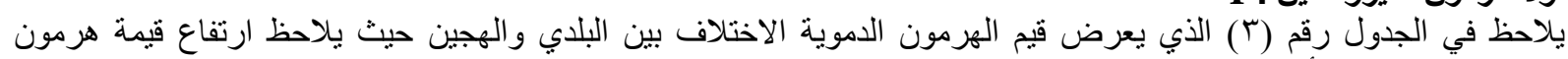

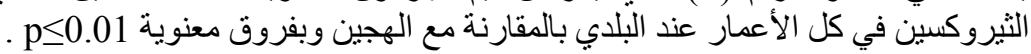


حيث بلغت قيمة هرمون الثيروكسين عند البلدي في الأسبوع الأول (اس. • (1) نانو غرام/مل وعند الهجين (0. ج) نانو غر ام/مل ولوحظ

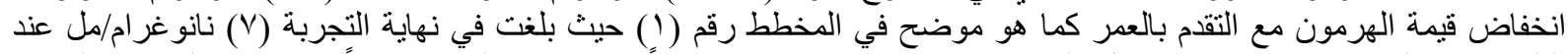

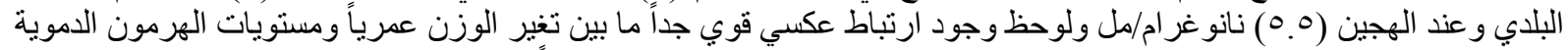

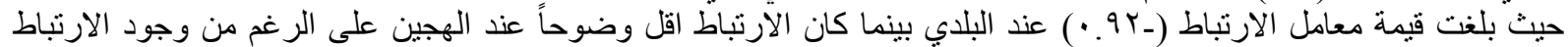

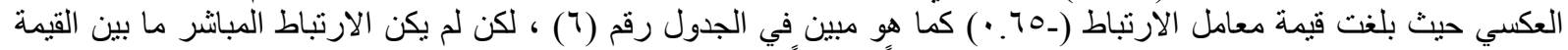

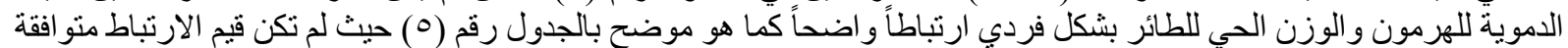
خلال اسابيع التربية.

ثانياً هرمون ثالث يود الثيرونين T3

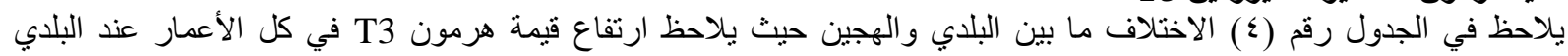

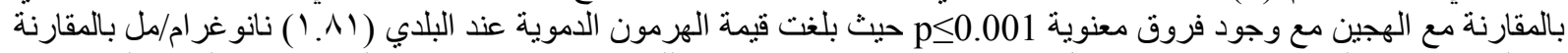

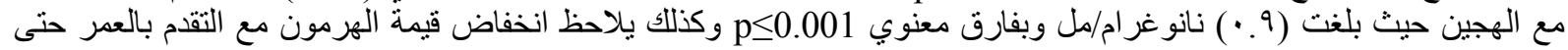

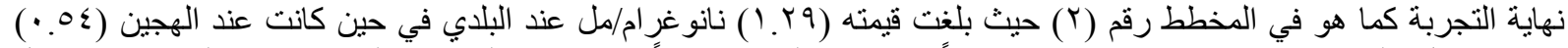

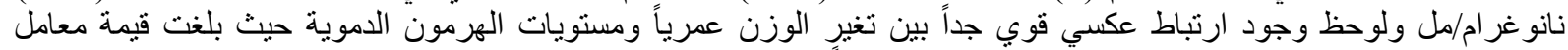

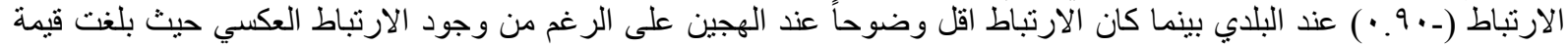

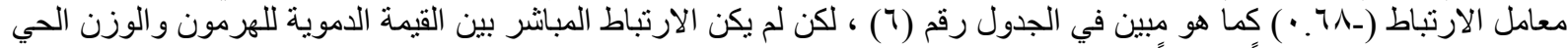

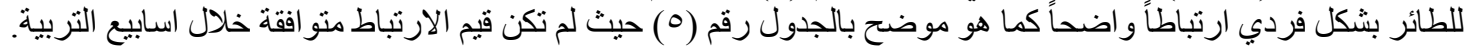

جدول رقم 1: الخلطات العلفية الأساسية المستخدمة في التجربة

\begin{tabular}{|c|c|c|}
\hline $\begin{array}{l}\text { ثانية من Y Y Y } \\
\text { \% }\end{array}$ & مرحلة أولى من 1-1 & المادة العلفية \\
\hline $0 \leqslant . M Y$ & 87.1 & ذزرة صفراء \\
\hline$r 0.0$ & $\varepsilon Y . V$. & كسبة صويا ؟ ؛ \% \\
\hline 7.1 & $V . Y$ & زيت نباتي \\
\hline 1.50 & 1.1 & فوسفات ثنائية الكالسيوم \\
\hline$\because \cdot 1$ &. .19 & ميثيونين \\
\hline .1 & .1 & كلوريد كولين \\
\hline $1 . r$ & $1 . Y T$ & كريونات الكالسيوم \\
\hline.. $\mathrm{ro}$ &..$\leqslant \wedge$ & ملح طعام \\
\hline$\because .0$ & $\because .0$ & مضاد فطور \\
\hline$\because .0$ & $\because .0$ & مضاد ـ. كوكسيديا \\
\hline. .1 & .1 & فيتامين+معادن* \\
\hline $1 \ldots$ & $1 \ldots$ & المجموع \\
\hline
\end{tabular}

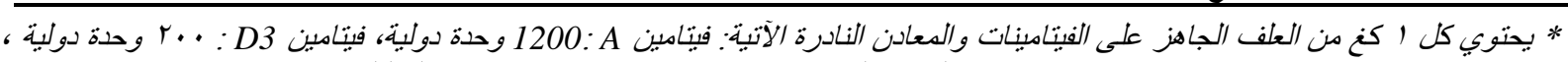

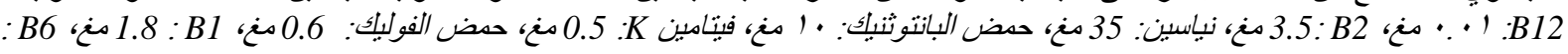

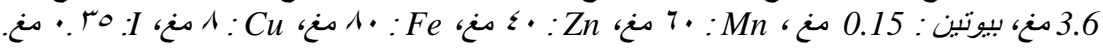

جدول رقم ץ : تحليل الخلطات العلفية وقيمتها الغذائية المحسوبة

\begin{tabular}{|c|c|c|}
\hline مرحلة ثانية & مرحلة أولى ألى & المكونات الغذائية \\
\hline$r r \leq q$ & rIVr & طاقة قابلة للتمثيل ك.ك/كغ \\
\hline$r \cdot . r q$ & $r$ r.Ar & بروتين \% \\
\hline 109.99 & $1 \% \wedge .9 \leq$ & $* \mathbf{C} / \mathbf{P}$ \\
\hline 1.99 & $1 . r V$ & لايسين \% \\
\hline$\because \varepsilon \cdot$ & $\because 0 \leqslant$ & ميثيونين\% \\
\hline.$V r$ & $\because 9$ & ميثيونين + سيستين\% \\
\hline$\because 9$. & 1 & كالسيوم\% \\
\hline.$r v$ &. .87 & فوسفور متاح\% \\
\hline .10 & $\cdot r$ & صوديوم \% \\
\hline. .47 & 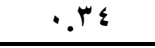 & كلور\% \\
\hline$\varepsilon . \vee 9$ & $\varepsilon . \wedge \mathrm{V}$ & حمض اللينوليك \\
\hline$r .71$ & $\varepsilon .1$ & ألياف خام \\
\hline
\end{tabular}


جدول رقم بّ: قيم هرمون الثيروكسين (T4) نانو غر ام/مل دم

\begin{tabular}{|c|c|c|}
\hline الهجين نانوغرام/مل & البلدي نانوغرام/مل & العمر \\
\hline $6.5 \pm 0.5$ & $10.31 \pm 1.42$ & $\mathrm{~V}$ \\
\hline $7.9 \pm 0.62$ & $8.8 \pm 0.30$ & $1 \varepsilon$ \\
\hline $7.5 \pm 0.73$ & $8.6 \pm 0.41$ & YI \\
\hline $6.7 \pm 0.11$ & $8.3 \pm 0.60$ & $r \wedge$ \\
\hline $6.9 \pm 0.54$ & $8.0 \pm 0.40$ & ro \\
\hline $5.5 \pm 0.13$ & $7.0 \pm 0.10$ & $\varepsilon Y$ \\
\hline
\end{tabular}

الجدول رقم ؛ : قيم هرمون ثالث يود التيرونين (T3) نانو غرام/مل دم

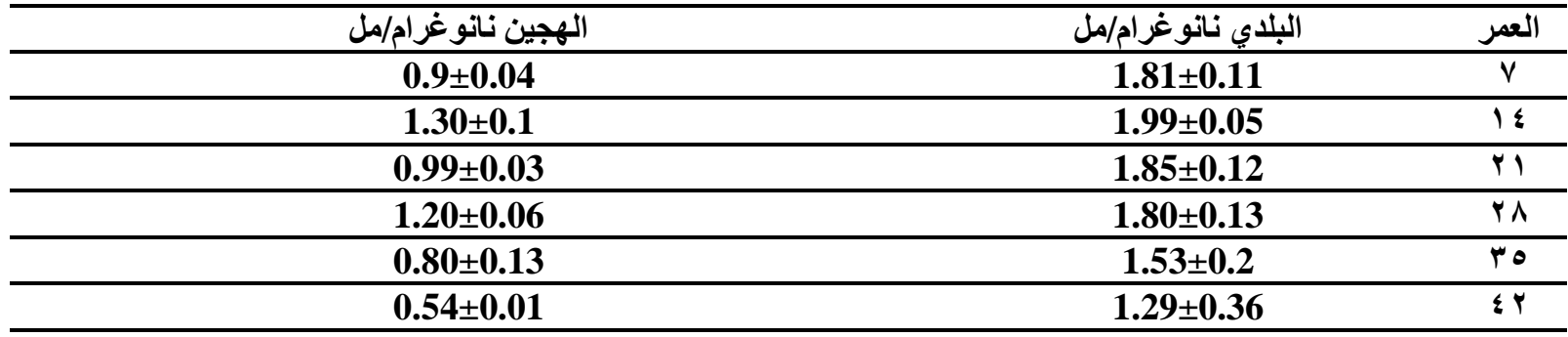

الجدول رقم ه: معاملات الارتباط ما بين القيمة الدموية للهرمون ووزنه الحي.

\begin{tabular}{|c|c|c|c|c|c|c|c|}
\hline الهجين العمر & الوزن غ & T3 & T4 & البلدي العمر & الوزن غ & T3 & T4 \\
\hline $\mathrm{V}$ & $200 \pm 14$ & -0.54 & 0.47 & 1 & $50 \pm 6$ & 0.17 & -0.45 \\
\hline $1 \varepsilon$ & $440 \pm 38$ & -0.4 & 0.34 & $1 \varepsilon$ & $85.8 \pm 12$ & -0.60 & -0.28 \\
\hline YI & $914 \pm 70$ & -0.15 & 0.09 & YI & $154 \pm 19$ & 0.53 & -0.39 \\
\hline rA & $1474 \pm 110$ & 0.60 & 0.06 & rA & $215 \pm 30$ & -0.58 & -0.26 \\
\hline ro & $2100 \pm 140$ & -0.19 & 0.22 & ro & $288 \pm 45$ & -0.64 & -0.19 \\
\hline$\varepsilon Y$ & $2681 \pm 237$ & -0.69 & 0.17 & $\varepsilon Y$ & $435 \pm 23$ & 0.83 & -0.27 \\
\hline
\end{tabular}

الجدول :6 معامل الارتباط العمودي (الأسابيع و الوزن و القيمة الدموية للهرمون)

\begin{tabular}{lcc}
\hline & T3 & T4 \\
\hline الهجين & -0.68 & -0.65 \\
\hline البلاين & -0.92 & -0.92 \\
\hline
\end{tabular}

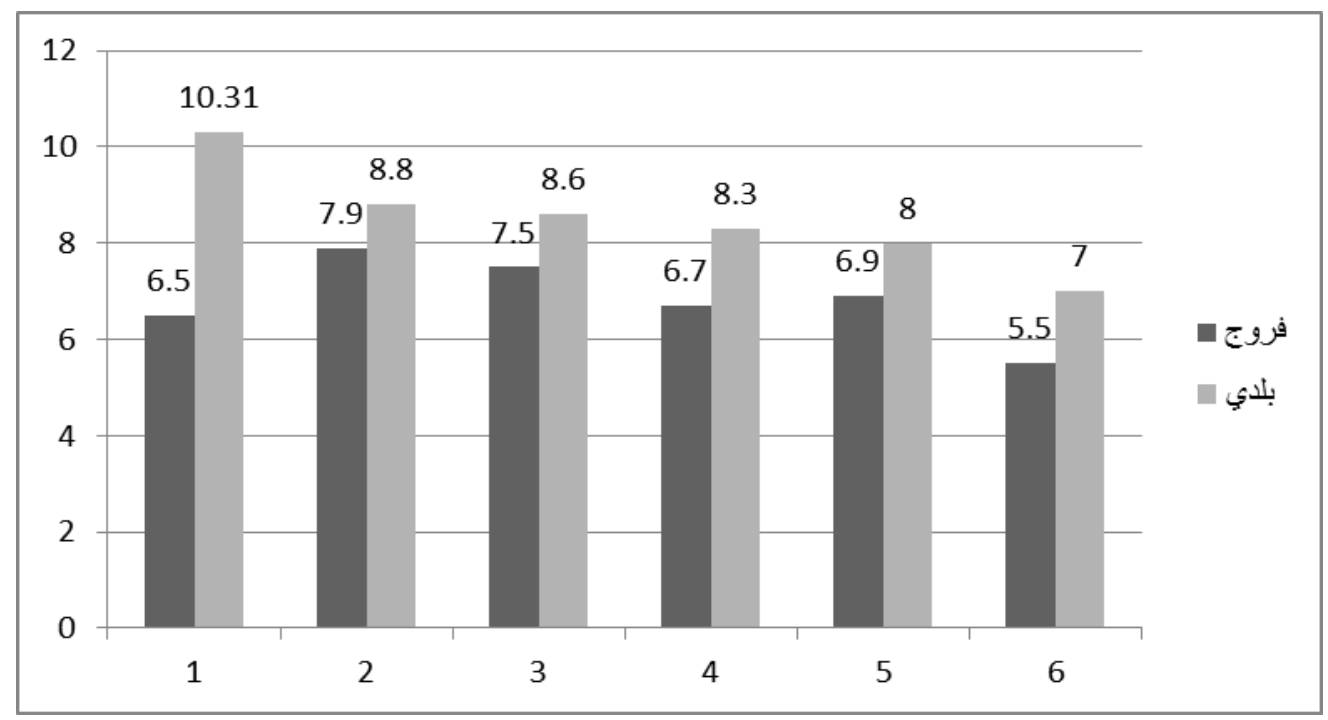

مخطط رقم 1: مستويات هرمون الثيروكسين نانو غر ام/مل خلال التجربة 


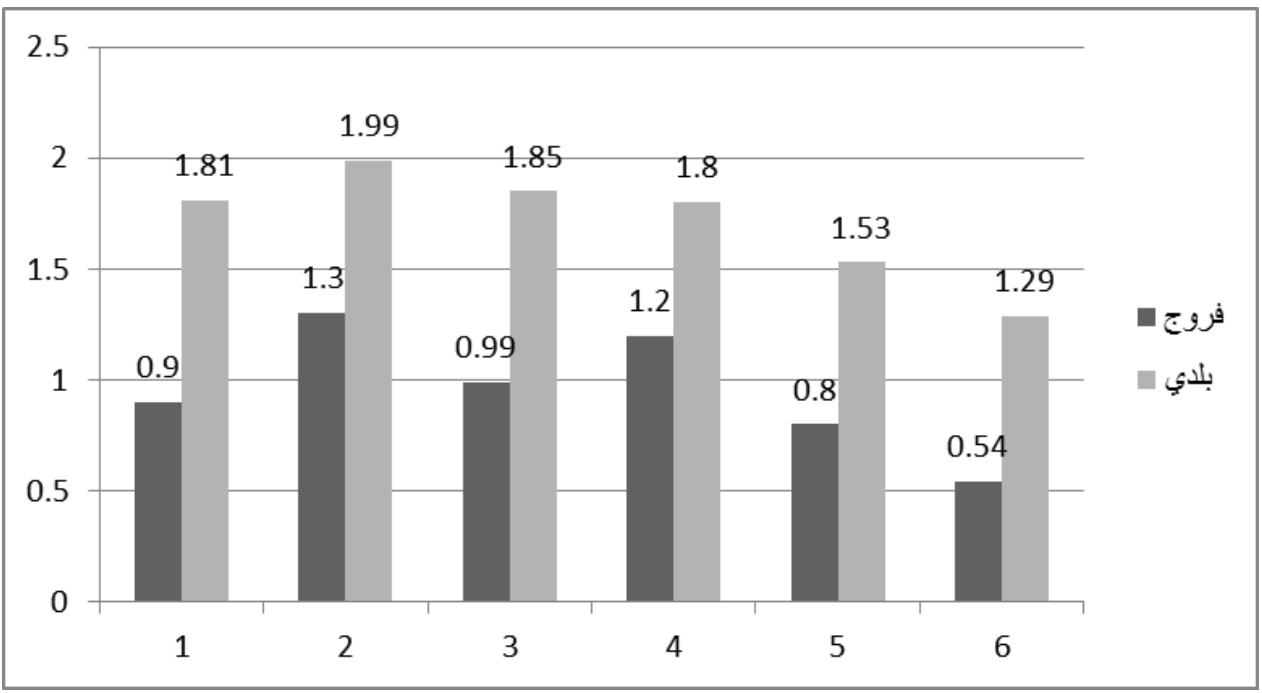

مخطط رقم ץ: مستويات هرمون ثالث يود الثيرونين T3 خلال التربية.

\section{DISCUSSION}

\section{المناقشة}

من أجل الحصول على المعايرة الحقيقية بين الأنواع الطبرية فإنه يلزم رعايتها في نفس الظروف البيئية وأن تحلل عينات البلازما في

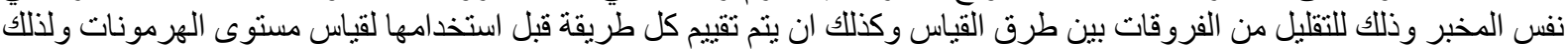

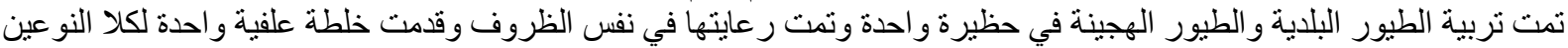

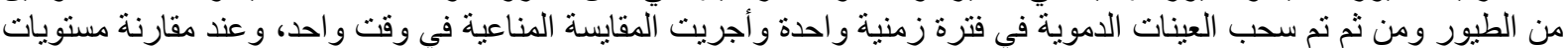

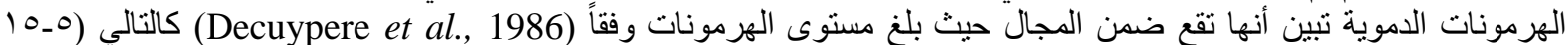

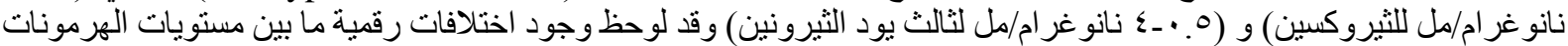
حسب الطريقة المعتمدة من قبل الباحث واختلافات اخرى نابعة عن سلالة الطيور ونوع التربية والتغذية ومحتوى اليود

.(Sturkie,2000)

لوحظ ارتفاع المستويات الهرمونية عند البلدي بالدقارنة مع الهجين في التجربة الحالية في جميع الأعمار حيث بين (Sturkie,1965)

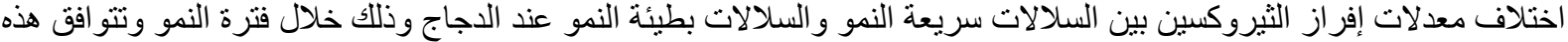

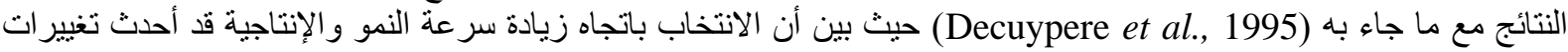
هرمونية استقلابية عند الهجن انتاج اللحم الحديثة.

ويمكن ايضاح هذه الملاحظة وتفسير ها بناءً على دور هرمونات الدرق في الاستقلاب الكلي في الجسم حيث ثبت بالتجارب دوره في

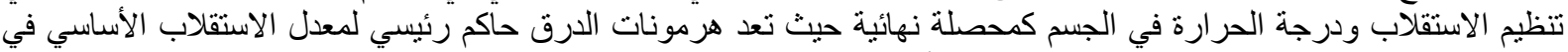

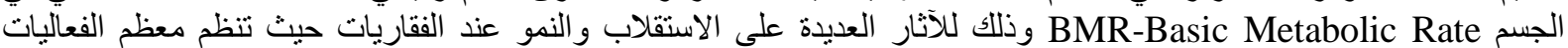

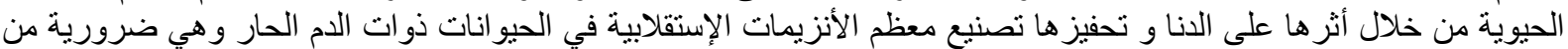

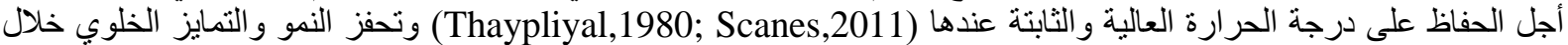

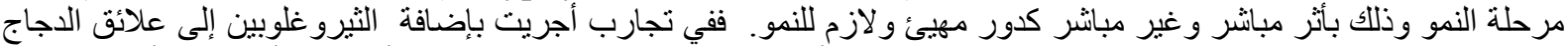

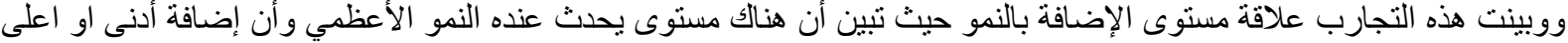

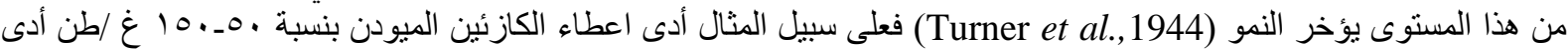

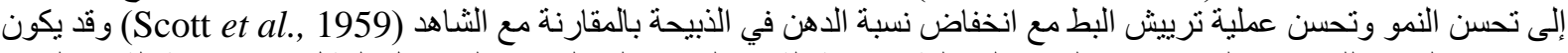

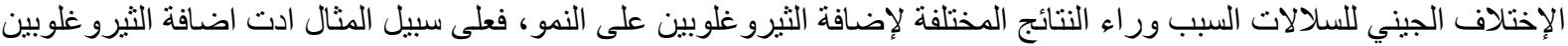

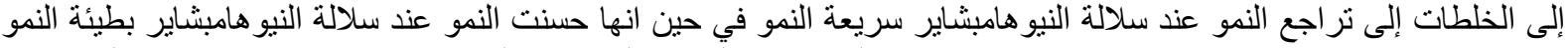

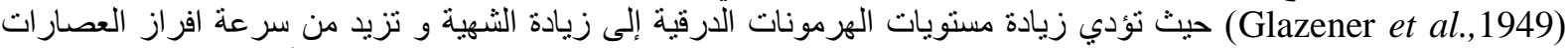

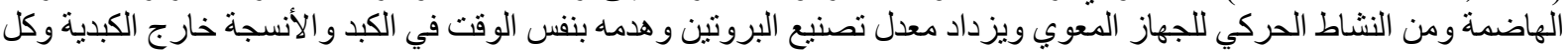

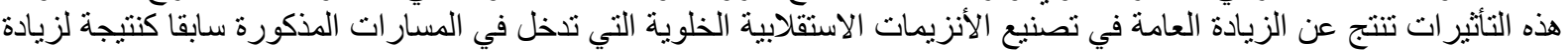

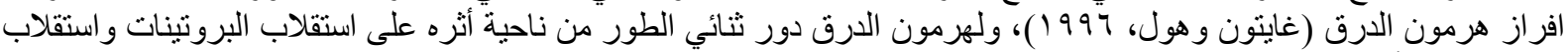

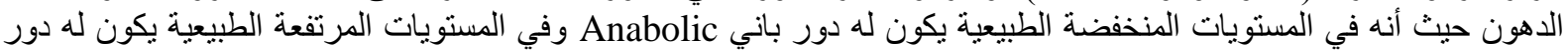

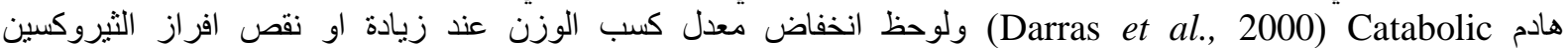

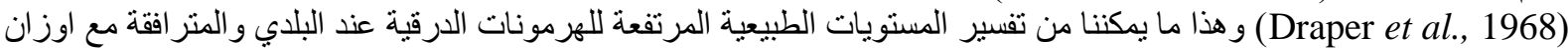

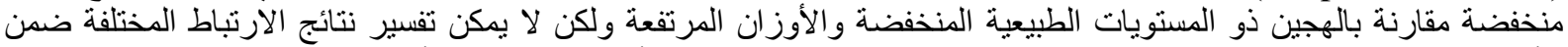
الأسابيع بين القيمة الدموية للهرمون و الوزن الحي و على ما يبدو هنالك عو امل أخرى تحكم هذا الأمر وتحتاج إلى مزيد من الأرئ الدراسات. 
وقد تم ابراز دور هرمونات الدرق في النمو عند الطيور من خلال ملاحظة التراجع الواضح في النمو الناتج عن استئصسال الغدة

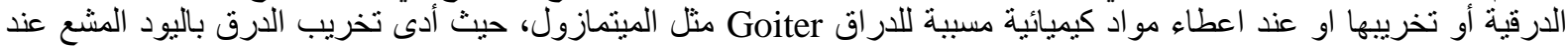

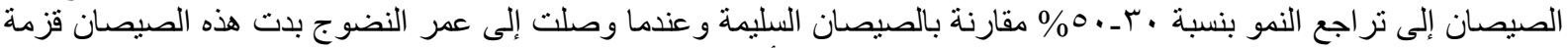

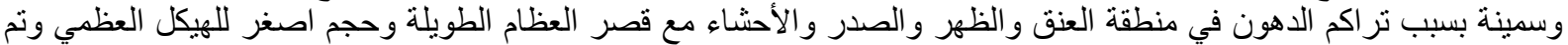

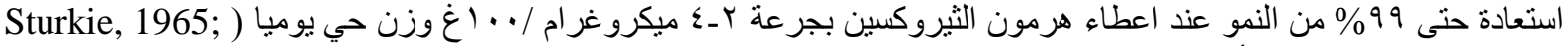

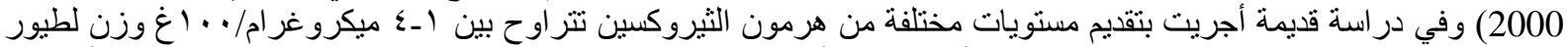

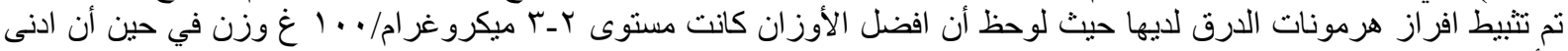

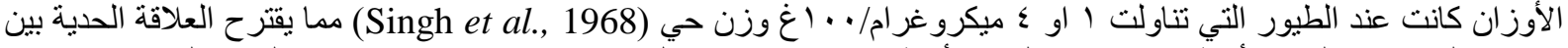

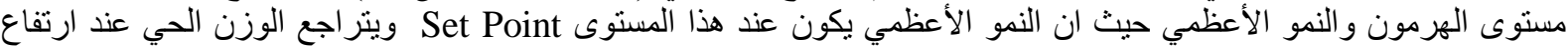
المستوى او انخفاضه عن هذا المستوى Set Point، وتختلف قيمة هذا المستوى بحسب السلالة للطائر كما ظهر بالنتائج آلحالية.

وتتخفض مستويات الهرمونات الدرقية الدموية مع التقام بالعمر عند الطيور وتتخفض بشكل خطي تقريبا وهذا الأمر مشابه لما يحدث

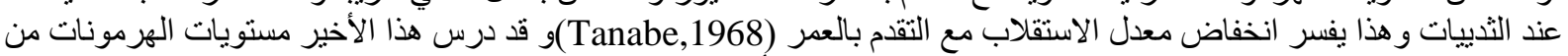

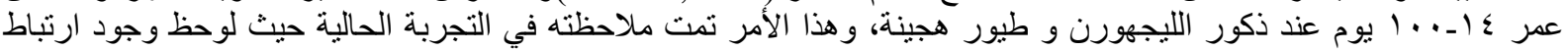

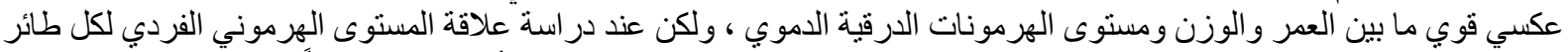

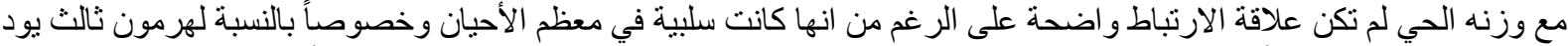

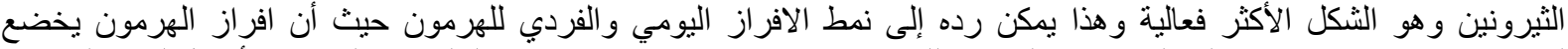

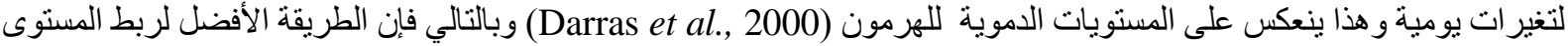

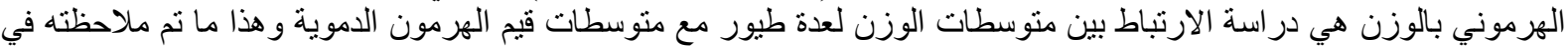

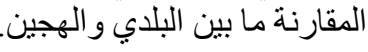

\section{الاستنتاجات \\ CONCLUSIONS}

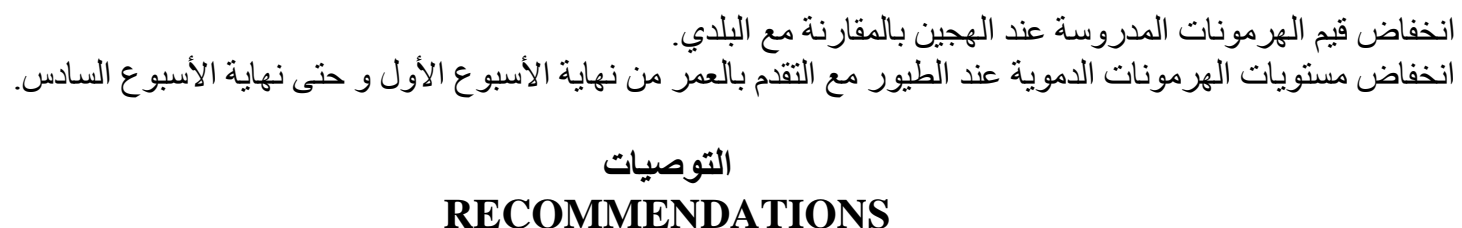
RECOMMENDATIONS

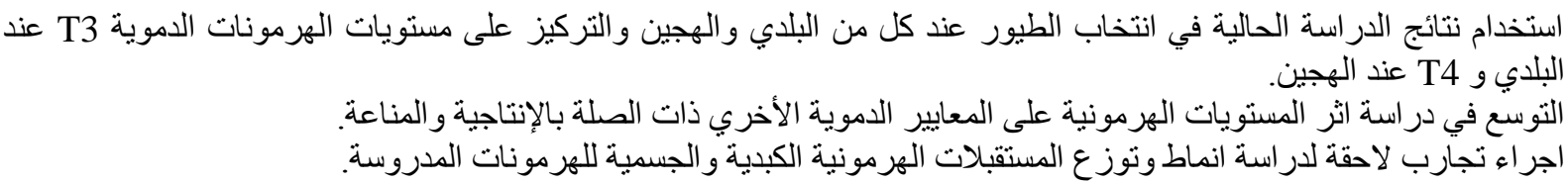

\section{REFERENCES}

Darras, S. Van der Geyten and Kühn, ER. (2000): Thyroid hormone metabolism in poultry. Biotechnol. Agron. Soc. Environ. 4 (1), 13-20.

Decuypere, E.; Buyse, J.; Rahimi, G. and Zeman, M. (1995): Comparative study of endocrinological parameters in the genetic lines of broilers. An overview. OECD Work shop "Growth and quality in broiler production". Arch. Geflu" gelkd. Sonderheft: 6-8.

Decuypere, J.; Buyse, C.; Scanes, G.; Huybrechts, L. and Kuhen, E.R. (1986): Effects of hyper- or hypothyroid status on growth, adiposity and levels of growth hormone, somatomedin $\mathrm{C}$ and thyroid metabolism in broiler chickens Reprod. Nutr. Develop., 555-565.

Draper, S.A.; Falconer, I.R. and Lamming, G.E. (1968): J. Physiol., Lond. 197, 659-665. IN Physiology and Biochemistry of the Domestic Fowl, Volume 1, Edited Bell D J, Freeman B. M 1971.

Glazener, E.W.; Shaffner, C.S. and Jull, M.A. (1949): Thyroid activity as related to strain differences on growing chickens. Poultry Sci. 28.834.

Gonzales, E.; Buyse, J.; Sartori, J.R.; Loddi, M.M. and Decuypere, E. (1999): Metabolic Disturbances in Male Broilers of Different Strains 2. Relationship Between the Thyroid and Somatotropic Axes with Growth Rate and Mortality, Poultry Science 78:516-521. 
Office International des Epizooties OIE (2000): Manual of Standards for Diagnostic Tests and Vaccines.

Ritchie, B.W.; Harrison, G.J. and Harrison, L.R. (1994): Avian Medicine :Principles and Application, .Winger Publishing Inc , Lake worth, Florida.

Scanes, C.G. (2011): Hormones and Metabolism in Poultry, Update on Mechanisms of Hormone Action -Focus on Metabolism, Growth and Reproduction, Prof. Gianluca Aimaretti (Ed.), ISBN: 978-953-307-341-5.

Scott, M.L.; Baker, R.C. and Dougherty, E. (1959): Iodinated casein in duck feed: preliminary finding feed illustrated. Aug. p53.

Singh, A.; Reineke, E.P. and Ringer, R.K. (1968): Pout. Sci. 47, 212-219. In Physiology and Biochemistry of the Domestic Fowl, Volume 1, Edited Bell D J, Freeman B. M 1971.

SPSS Inc. Released (1997): SPSS for Windows, Version 7.5. Chicago, SPSS Inc.

Sturkie, P.D. Avian Physiology, (1965): Second edition, Cornell University Press, New York pp 327356.

Sturkie, P.D. and Whittow, G.C. (2000): Sturkie's avian physiology - 5th ed.San Diego [etc.]: Academic Press.p 461-470.

Tanabe, $Y$. (1965): Relation of Thyroxine Secretion Rate to Age and Growth Rate in the Cockerel. Poultry Science (1965) 44 (2): 591-596.

Thaypliyal, J.P. (1980): Hormones, Adaptation and Evolution (S.Ishii et al., eds.), 241-250 Japan SciSoc Press, Tokyo/ Springer-Verlag, Berlin (1980).

Turner, CW.; Irwin, M.R. and Reineke, E.P. (1944): Effect of feeding thyroactiveiodocasein to Barred Rock cockerels. Poultry Sci.23:242.

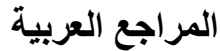

غايتون ، هول، (79 99 ) : المرجع في الفيزيولوجيا الطبية منشور ات منظمة الصحة العالمية، مكتب الثرق الأوسط. 